\title{
Uso de substâncias psicoativas entre estudantes de enfermagem
}

\author{
Psychoactive substance use between students of the nursing \\ Paula Silva Mardegan', Renata Santos de Souza', Vitor Buaiz', Marluce Miguel de Siqueira'
}

\section{RESUMO}

Objetivo: Traçar o perfil do uso de substâncias psicoativas entre os universitários do curso de Enfermagem do Centro de Ciências da Saúde da Universidade Federal do Espírito Santo. Métodos: Trata-se de um estudo exploratório, descritivo, transversal e quantitativo, desenvolvido com universitários de Enfermagem do primeiro ao último ano do curso. 0 instrumento utilizado na coleta de dados foi o "Questionário sobre o Uso de Droga", uma adaptação do proposto pela Organização Mundial de Saúde (OMS) e desenvolvido pela WHO - Research and Reporting Project on the Epidemiology of Drug Dependence. Os dados foram tabulados e analisados por meio do programa Statistical Packcage for the Social Science (SPSS, 2005). Resultados: Dos universitários, 82\% são do sexo feminino, 46,6\% se encontram na faixa etária de 20 a 22 anos e 41\% pertencem à classe social B. Quanto ao uso de substâncias psicoativas, 43,9\% fizeram uso na vida de alguma substância, exceto álcool e tabaco, 82,1\% relataram uso na vida de álcool, 11,7\% informaram uso freqüente e 6,2\% uso pesado dessa substância, e 22,4\% mencionaram uso na vida de tabaco. Conclusão: Fazse necessária a prevenção do uso indevido de substâncias psicoativas entre universitários, por meio de disciplinas curriculares que abordem a temática ou de programas específicos destinados a essa população.

Substâncias psicoativas, universitários, prevenção.

\section{ABSTRACT}

Objective: To trace the profile of the psychoactive substance use between the university students of the course of Nursing Sciences Health Center of the Federal University of Espirito Santo. Methods: One is about explorer, descriptive, transversal and quantitative study, developed with Nursing university students of the first to the last year of the course. The instrument used in the collection of data is the Questionnaire on the Use of Drug, an adaptation of the considered one for the OMS and developing for the WHO - Research and Reporting Project on the Epidemiology of 


\section{Key-words}

Psychoactive substances, university students, prevention.
Drug Dependence. The data had been tabulated and analyzed through the Statistical Packcage Program for the Social Science (SPSS, 2005). Results: $82 \%$ of the university students are female, $46.6 \%$ it had between 20 and 22 years and $41 \%$ belong to the social class B. Regarding the use of psychoactive substances, 43,9\% made use in the life of some substance, except alcohol and tobacco, 82,1\% reported use of alcohol in the life, $11,7 \%$ reported frequently used and 6,2\% heavy use of this substance; and 22,4\% mentioned in the life of tobacco use. Conclusion: It is necessary to prevent the misuse of psychoactive substances among university students through curriculum subjects that address the theme or specific programs for this population.

\section{INTRODUÇÃO}

O consumo de substâncias psicoativas tem aumentado significativamente nos últimos anos, sendo o início do uso cada vez mais precoce. Atualmente, há uma preocupação deste uso entre os estudantes, entre eles, os universitários.

O comportamento dos universitários diante das substâncias psicoativas já foi alvo de diversos estudos envolvendo universidades do Brasil inteiro, dos quais ressaltamos pesquisas realizadas na Universidade de São Paulo (KerrCorrea et al., 1999), na Universidade Estadual Paulista (Balan e Campos, 2006), na Universidade do Estado do Rio de Janeiro (Matsumoto et al., 2005), na Universidade Federal do Amazonas (Lucas et al., 2006), na Universidade Federal de Goiás (Canuto et al., 2006), na Universidade Federal do Ceará (Souza et al., 1999), na Universidade Estadual de Feira de Santana (Alves et al., 2005). Porém, apesar desta diversidade de pesquisas, é importante o desenvolvimento de novos estudos voltados para essa temática, pois serão seus resultados que auxiliarão no aumento da conscientização sobre o problema do uso de drogas e os fatores a ele associados, bem como subsidiarão a criação de programas de prevenção voltados a essa população.

Neste sentido, o objetivo do estudo é traçar o perfil do uso de substâncias psicoativas entre acadêmicos do curso de graduação em Enfermagem do Centro de Ciências da Saúde da Universidade Federal do Espírito Santo (CCS-Ufes).

\section{MÉTODOS}

A pesquisa foi realizada no curso de Enfermagem do CCSUfes, no período de janeiro a junho de 2007. A população do estudo foi constituída de alunos matriculados no referido curso nos oito períodos acadêmicos, totalizando 239 alunos. A amostra foi calculada no software Epi Info 6.04, considerando-se o grau de confiabilidade de 95\%, a precisão de $2 \%$ e a prevalência de "usuários de droga na vida" de 10\%, obtendo-se uma amostra de 188 estudantes. Entretanto, dos 239 alunos matriculados no curso de Enfermagem, $60(25,1 \%)$ não estavam presentes em sala de aula no mo- mento da aplicação do questionário ou, apesar de presentes, se recusaram a participar da pesquisa. Sendo assim, a amostra final foi constituída de 179 estudantes.

A pesquisa foi aprovada pelo Comitê de Ética em Pesquisa do CCS-Ufes por meio do Processo no 104/2006. Inicialmente foi enviada uma carta ao chefe de departamento do curso de Enfermagem, solicitando a colaboração dos professores na concessão de alguns minutos de sua aula para a aplicação dos questionários. Foi utilizado um questionário fechado, de autopreenchimento e sem identificação pessoal do aluno, aplicado a todos os alunos presentes em sala de aula no momento da pesquisa. Não era obrigatória a participação, porém todos que aceitaram participar da pesquisa assinaram o Termo de Consentimento Livre e Esclarecido, de acordo com a Resolução no 196/1996 do Conselho Nacional de Saúde. O instrumento utilizado foi o "Questionário sobre o Uso de Droga", uma adaptação do instrumento proposto pela Organização Mundial de Saúde (OMS) e desenvolvido pela WHO - Research and Reporting Project on the Epidemiology of Drug Dependence (Smart et al., 1980). No Brasil, este instrumento foi adaptado por Carlini-Cotrim et al. (1989), sendo também utilizado nos levantamentos nacionais sobre o uso de drogas em estudantes de primeiro e segundo graus, realizados pelo Centro Brasileiro de Informações sobre Drogas Psicotrópicas (Cebrid) em 1987, 1989, 1993, 1997 e 2004.

O instrumento é dividido em cinco partes, porquanto: (a) a primeira parte explica a pesquisa convidando o estudante a dela participar; (b) a segunda parte é compreendida por questões referentes a dados sociodemográficos; (c) a terceira parte contempla questões envolvendo o uso de substâncias psicoativas, incluindo tabaco, álcool, maconha, cocaína e derivados, medicamentos anfetamínicos, ansiolíticos, anticolinérgicos, orexígenos, barbitúricos, opióides, xaropes à base de codeína, solventes, alucinógenos, além de questões sobre o uso de anabolizantes, que foram incluídas no estudo pelo seu significativo abuso, embora não sejam drogas psicoativas, bem como questões sobre o uso de drogas injetáveis; (d) a quarta parte consiste de questionamento minucioso sobre o uso abusivo de álcool; e (e) a quinta parte é constituída por um questionamento 
sobre o relacionamento entre os pais e os estudantes entrevistados. As variáveis socioeconômicas foram avaliadas segundo critérios da Associação Brasileira do Instituto de Mercado e Pesquisa (Abipeme, 1978).

Foi realizado uma aplicação-piloto do instrumento de coleta de dados em três alunos matriculados no 10, $5^{\circ}$ e $8^{\circ}$ períodos do curso de Enfermagem, para capacitação do pesquisador e adequação do instrumento, sendo necessário ajustar a questão 36 para melhorar sua compreensão.

Os dados foram analisados pelo programa Statistical Package for the Social Science (SPSS), 2005, visto que os dados referentes ao consumo de drogas pelos universitários foram analisados de acordo com os indicadores de classificação sobre o uso de drogas, preconizados pela OMS (1980): "uso na vida" (quando a pessoa fez uso pelo menos uma vez na vida); "uso no ano" (quando a pessoa fez uso pelo menos uma vez nos 12 meses que antecederam a pesquisa); "uso no mês" (quando a pessoa fez uso pelo menos uma vez nos 30 dias que antecederam a pesquisa); "uso freqüente" (quando a pessoa fez uso seis ou mais vezes nos 30 dias que antecederam a pesquisa); e "uso pesado" (quando a pessoa fez uso 20 ou mais vezes nos 30 dias que antecederam a pesquisa).

\section{RESULTADOS}

A Tabela 1 apresenta o perfil sociodemográfico dos universitários, no qual se observa que $82,7 \%$ dos estudantes pertenciam ao sexo feminino, houve predomínio da faixa etária entre 20 e 22 anos (47,5\%) e prevalência de alunos nas classes sociais B (41,3\%) e C (33\%). Quanto às faltas na universidade nos últimos 30 dias que antecederam à pesquisa, $41,9 \%$ dos alunos disseram não ter faltado, enquanto 35,7\% afirmaram ter faltado de um a três dias.

Na Tabela 2, que demonstra o uso na vida das substâncias psicoativas, observa-se prevalência elevada para o álcool (82,1\%), seguido do tabaco (22,3\%), dos ansiolíticos (13,4\%), dos anfetamínicos (11,7\%), dos solventes (11,2\%) e da maconha (6,7\%), enquanto os barbitúricos, os anticolinérgicos, os alucinógenos e a cocaína corresponderam a 0,6\%. Na categoria "uso no ano", houve redução da prevalência das substâncias, exceto os barbitúricos e os anticolinérgicos que se mantiveram cada um com 0,6\% da amostra. Quanto ao uso freqüente, houve relato apenas de substâncias lícitas, 11,7\% de estudantes relatando fazer uso de álcool e 1,7\% de tabaco. Já em relação ao uso pesado, foi relatado o uso de substâncias ilícitas, como os anfetamínicos, os solventes e a maconha correspondendo a 0,6\% da amostra, além do uso de álcool $(6,2 \%)$ e do tabaco (1,1\%).

A Tabela 3 demonstra a relação entre o sexo e o uso na vida de substâncias psicoativas entre universitários, na qual se observa uma diferença de consumo significativa entre os sexos (exceto o álcool), pois 93,1\% dos homens relataram
Tabela 1. Características sociodemográficas dos estudantes do curso de Enfermagem do CCS-Ufes, Vitória, 2007

\begin{tabular}{|c|c|c|}
\hline Características & $\mathrm{N}$ & $\%$ \\
\hline \multicolumn{3}{|l|}{ Sexo } \\
\hline Masculino & 29 & 16,2 \\
\hline Feminino & 148 & 82,7 \\
\hline Não informado & 2 & 1,1 \\
\hline Total & 179 & 100 \\
\hline \multicolumn{3}{|c|}{ Faixa etária (em anos) } \\
\hline 17 a 19 & 27 & 15,1 \\
\hline 20 a 22 & 85 & 47,5 \\
\hline 23 a 25 & 50 & 27,9 \\
\hline 26 a 28 & 11 & 6,1 \\
\hline$\geq 29$ & 3 & 1,7 \\
\hline Não informado & 3 & 1,7 \\
\hline Total & 179 & 100 \\
\hline \multicolumn{3}{|c|}{ Defasagem da Universidade } \\
\hline Nenhuma falta & 75 & 41,9 \\
\hline 1 a 3 faltas & 64 & 35,7 \\
\hline 4 a 8 faltas & 27 & 15,1 \\
\hline 9 ou mais faltas & 10 & 5,6 \\
\hline Não informado & 3 & 1,7 \\
\hline Total & 179 & 100 \\
\hline \multicolumn{3}{|c|}{ Nível socioeconômico } \\
\hline A & 36 & 20,1 \\
\hline B & 74 & 41,3 \\
\hline C & 59 & 33 \\
\hline$D$ & 9 & 5 \\
\hline $\mathrm{E}$ & 0 & 0 \\
\hline Não informado & 1 & 0,6 \\
\hline Total & 179 & 100 \\
\hline
\end{tabular}

Tabela 2. Uso de substâncias psicoativas (na vida, no ano, no mês, freqüente e pesado) entre estudantes do curso de Enfermagem do CCS-Ufes, Vitória, 2007

\begin{tabular}{|c|c|c|c|c|c|}
\hline \multirow{2}{*}{ Drogas } & Na vida & No ano & No mês & Freqüente & Pesado \\
\hline & $\%$ & $\%$ & $\%$ & $\%$ & $\%$ \\
\hline Álcool & 82,1 & 72,6 & 32,4 & 11,7 & 6,2 \\
\hline Tabaco & 22,3 & 9,5 & 2,2 & 1,7 & 1,1 \\
\hline Ansiolíticos & 13,4 & 9,5 & 1,7 & 0 & 0 \\
\hline Anfetamínicos & 11,7 & 7,3 & 1,1 & 0 & 0,6 \\
\hline Solventes & 11,2 & 6,1 & 0,6 & 0 & 0,6 \\
\hline Maconha & 6,7 & 1,7 & 0,6 & 0 & 0,6 \\
\hline Barbitúricos & 0,6 & 0,6 & 0,6 & 0 & 0 \\
\hline Anticolinérgicos & 0,6 & 0,6 & 0 & 0 & 0 \\
\hline Cocaína & 0,6 & 0 & 0 & 0 & 0 \\
\hline Alucinógenos & 0,6 & $*$ & * & $*$ & * \\
\hline Opiáceos & 0 & * & * & * & * \\
\hline Xaropes & 0 & * & * & * & * \\
\hline Orexígenos & 0 & * & * & * & * \\
\hline
\end{tabular}

* Dados não colhidos.

ter experimentado o álcool e 80,4\% das mulheres também mencionaram o uso; enquanto o uso na vida do tabaco correspondeu a 41,3\% dos homens e 18,9\% das mulheres. O uso na vida de substâncias ilícitas também foi maior entre os homens do que entre as mulheres, exceto o de barbitúricos e de alucinógenos, que apresentaram freqüência de uso nas mulheres e nenhum relato de uso entre os homens. 
Tabela 3. Uso na vida de substâncias psicoativas entre os estudantes do curso de Enfermagem do CCS-Ufes (distribuição por sexo), Vitória, 2007

\begin{tabular}{|c|c|c|c|c|c|}
\hline \multirow{2}{*}{ Droga } & \multicolumn{2}{|c|}{ Masculino } & \multicolumn{2}{|c|}{ Feminino } & \multirow{2}{*}{$\frac{\text { Tota }}{\mathrm{N}}$} \\
\hline & $\mathrm{N}$ & $\%$ & $\mathrm{~N}$ & $\%$ & \\
\hline Álcool & 27 & 93,1 & 119 & 80,4 & 146 \\
\hline Tabaco & 12 & 41,3 & 28 & 18,9 & 40 \\
\hline Maconha & 6 & 20,7 & 6 & 4,1 & 12 \\
\hline Cocaína & 1 & 3,4 & 0 & 0 & 1 \\
\hline Anfetamínicos & 7 & 24,1 & 14 & 9,5 & 21 \\
\hline Solventes & 4 & 13,8 & 16 & 10,8 & 20 \\
\hline Ansiolíticos & 4 & 13,8 & 20 & 13,5 & 24 \\
\hline Anticolinérgicos & 1 & 3,4 & 0 & 0 & 1 \\
\hline Barbitúricos & 0 & 0 & 1 & 0,7 & 1 \\
\hline Alucinógenos & 0 & 0 & 1 & 0,7 & 1 \\
\hline
\end{tabular}

Em relação à idade de experimentação das substâncias psicoativas, a Tabela 4 demonstra que a idade de início para as substâncias lícitas predominou na faixa etária entre 16 e 18 anos, havendo relatos de uso anteriores aos 12 anos. Já, as substâncias ilícitas tiveram maior uso inicial entre 16 e 18 anos, para a maconha e os solventes, e acima de 18 anos, para os anfetamínicos e ansiolíticos.

A Tabela 5 apresenta as bebidas alcoólicas mais consumidas entre os estudantes de Enfermagem, a freqüência de consumo e os locais de uso, apontando o predomínio das bebidas fermentadas - cerveja, 32,9\% e vinho, 15,6\%, freqüência de uso de 1 a 5 vezes por semana $(32,4 \%)$ e consumo de 1 a 2 doses por vez (36,3\%). Os locais de uso mais referidos pelos universitários foram os bares, as danceterias e as boates, correspondendo a 44,1\% da amostra, porquanto $50,8 \%$ dos estudantes relataram beber na companhia de amigos.

De acordo com a Tabela 6, 21,2\% dos estudantes relataram a ocorrência de algum evento após o uso de bebidas alcoólicas, e desses, 6,1\% se envolveram em brigas, 2,8\% já sofreram acidentes, 4,4\% já dirigiram, 7,3\% já faltaram à faculdade e 0,6\% já faltaram ao trabalho.
Tabela 5. Características dos estudantes do curso de Enfermagem do CCS-Ufes (em relação ao consumo de bebidas alcoólicas), Vitória, 2007

\begin{tabular}{|c|c|c|}
\hline \multirow{2}{*}{ Variáveis } & \multicolumn{2}{|c|}{ Uso no mês } \\
\hline & $\mathrm{N}$ & $\%$ \\
\hline \multicolumn{3}{|l|}{ Bebidas consumidas } \\
\hline Cerveja & 59 & 32,9 \\
\hline Vinho & 28 & 15,6 \\
\hline Vodka & 12 & 6,7 \\
\hline Pinga & 4 & 2,2 \\
\hline Sidra & 1 & 0,6 \\
\hline Outras & 6 & 3,4 \\
\hline \multicolumn{3}{|l|}{ Doses consumidas } \\
\hline 1 a 2 doses & 65 & 36,3 \\
\hline 3 a 4 doses & 35 & 19,6 \\
\hline 5 a 6 doses & 12 & 6,7 \\
\hline 7 a 9 doses & 4 & 2,3 \\
\hline 10 ou mais doses & 4 & 2,2 \\
\hline \multicolumn{3}{|l|}{ Freqüência de uso } \\
\hline 1 a 5 vezes & 58 & 32,4 \\
\hline 6 a 19 vezes & 21 & 11,7 \\
\hline 20 vezes ou mais & 11 & 6,2 \\
\hline \multicolumn{3}{|l|}{ Local de uso } \\
\hline Em casa & 31 & 17,3 \\
\hline Bares/danceterias/boates & 79 & 44,1 \\
\hline Casa de amigos & 22 & 12,3 \\
\hline \multicolumn{3}{|c|}{ Pessoas com as quais costumam beber } \\
\hline Familiares & 23 & 12,8 \\
\hline Amigos & 91 & 50,8 \\
\hline Outros & 2 & 1,1 \\
\hline
\end{tabular}

Tabela 6. Eventos ocorridos após beber em estudantes do curso de Enfermagem do CCS-Ufes, Vitória, 2007

\begin{tabular}{lrc}
\hline Eventos ocorridos & N & $\%$ \\
\hline Brigou & 11 & 6,1 \\
Sofreu acidentes & 5 & 2,8 \\
Dirigiu & 8 & 4,4 \\
Faltou à faculdade & 13 & 7,3 \\
Faltou ao trabalho & 1 & 0,6 \\
Nenhum & 141 & 78,8 \\
Total & 179 & 100 \\
\hline
\end{tabular}

Tabela 4. Uso de substâncias psicoativas entre estudantes de Enfermagem do CCS-Ufes (idade de experimentação), Vitória, 2007

\begin{tabular}{|c|c|c|c|c|c|c|c|c|c|c|c|c|c|}
\hline \multirow[t]{2}{*}{ Droga } & \multicolumn{2}{|c|}{ Abaixo de 10 anos } & \multicolumn{2}{|c|}{ 10-12 anos } & \multicolumn{2}{|c|}{ 13-15anos } & \multicolumn{2}{|c|}{$16-18$ anos } & \multicolumn{2}{|c|}{ Acima de 18 anos } & \multicolumn{2}{|c|}{ Não lembra } & \multirow[t]{2}{*}{ Total } \\
\hline & $\mathrm{N}$ & $\%$ & $\mathrm{~N}$ & $\%$ & $\mathrm{~N}$ & $\%$ & $\mathrm{~N}$ & $\%$ & $\mathrm{~N}$ & $\%$ & $\mathrm{~N}$ & $\%$ & \\
\hline Maconha & 0 & 0 & 0 & 0 & 0 & 0 & 7 & 3,9 & 2 & 1,2 & 3 & 1,6 & 12 \\
\hline Anfetamínicos & 0 & 0 & 0 & 0 & 2 & 1,1 & 5 & 2,8 & 14 & 7,9 & 1 & 0,6 & 22 \\
\hline Solventes & 0 & 0 & 0 & 0 & 4 & 2,2 & 6 & 3,4 & 6 & 3,4 & 4 & 2,2 & 20 \\
\hline Ansiolíticos & 0 & 0 & 0 & 0 & 1 & 0,6 & 5 & 2,8 & 10 & 5,6 & 8 & 4,5 & 24 \\
\hline Barbitúricos & 0 & 0 & 1 & 0,6 & 0 & 0 & 0 & 0 & 0 & 0 & 0 & 0 & 1 \\
\hline Tabaco & 1 & 0,6 & 2 & 1,1 & 11 & 6,1 & 13 & 7,3 & 9 & 5,1 & 4 & 2,2 & 40 \\
\hline Álcool & 1 & 0,6 & 9 & 5 & 33 & 18,4 & 46 & 25,7 & 8 & 4,5 & 50 & 27,9 & 147 \\
\hline
\end{tabular}

\section{DISCUSSÃO}

A amostra deste estudo é composta por uma porcentagem muito maior de universitários do sexo feminino (82,7\%) do que do masculino, estando de acordo com os achados de
Marçal et al. (2005), em sua pesquisa realizada entre estudantes de Enfermagem, que encontraram 88\% de alunos pertencentes ao sexo feminino. Isso reflete a cultura que a Enfermagem possui, desde o seu surgimento, de ser uma profissão predominantemente feminina. 
Nossos achados demonstraram que a maioria dos estudantes (82,1\%) já fez uso na vida de álcool, dado semeIhante ao encontrado por Kerr-Correa et al. (1999), que detectou a prevalência de $84 \%$ para o uso na vida dessa substância entre estudantes de Medicina da Universidade Estadual Paulista (Unesp). O uso no ano de álcool (72,6\%) se assemelha aos resultados de Souza et al. (1999), que identificaram a prevalência de 79,6\% entre estudantes de Medicina. Já, o uso no mês (32,4\%) difere deste estudo, pois este obteve prevalência mais elevada, de 65,5\%, enquanto Silva et al. (2006) detectaram prevalência de 43,3\% entre estudantes universitários da área de ciências biológicas. O uso freqüente de álcool $(11,7 \%)$ encontrado neste estudo corrobora com os achados de Tavares et al. (2001), que detectaram uma prevalência de 16,8\% em um estudo com adolescentes escolares. Dos 179 universitários pesquisados, $6,2 \%$ relataram fazer uso pesado de álcool, dado divergente dos encontrados por Soldera et al. (2004), que obtiveram a prevalência de 11,9\% entre escolares, mas semelhante ao de Carvalho et al. (2000), que encontraram prevalência de 6,5\% entre universitários de ciências jurídicas e econômicas. Tais resultados são preocupantes, visto que o uso pesado de álcool pode levar a instalação da dependência. Segundo o Ministério da Saúde (apud Marçal, 2005, p. 10), "[...] essa pode ser caracterizada por perda da capacidade de controlar a ingestão, ênfase no consumo, tolerância, síndrome de abstinência e consumo de substâncias para aliviar os sintomas de abstinência".

Estudos realizados anteriormente sobre o uso de drogas psicotrópicas no Brasil mostraram que a faixa etária em que aparecem as maiores porcentagens de dependentes se encontra entre 18 e 24 anos (Carlini et al., 2001).

O estudo em questão apresentou para o tabaco prevalências de uso na vida (22,3\%), no ano (9,5\%), no mês (2,2\%), freqüente $(1,7 \%)$ e pesado $(1,1 \%)$ inferiores às encontradas por Galduróz et al. (1997) entre estudantes de 10 e $2^{\circ}$ graus em dez capitais brasileiras, no qual todas as capitais tiveram prevalências maiores que as encontradas neste estudo. Matsumoto et al. (2005) também encontraram uma prevalência de uso da na vida do tabaco mais elevada que a apresentada pelo presente estudo, 42\%, entre estudantes de Enfermagem da Universidade do Estado do Rio de Janeiro (Uerj). Essa diferença pode ser por causa da população em estudo ser composta por universitários de um curso da área da saúde que reflete no estudante a necessidade de ser exemplo para a sociedade.

O uso na vida de ansiolíticos (13,4\%) e anfetamínicos $(11,7 \%)$ encontrados neste estudo teve uma prevalência elevada comparado aos achados de Lucas et al. (2006), que encontraram 8,7\% e 5,98\%, respectivamente, em seu estudo envolvendo universitários da área da saúde, e Canuto et al. (2006) que detectaram prevalências de 7,7\% e 6,4\%, respectivamente. Já o uso na vida de maconha $(6,7 \%)$ e solventes $(11,2 \%)$ desta pesquisa difere de outras por ter uma prevalência reduzida. Silva et al. (2006), em uma pesquisa com escolares de ensino médio, detectaram prevalências de $12,1 \%$ e $18,1 \%$, respectivamente, e Carvalho et al. (2000) obtiveram resultados de 20,7\% e 27,5\%, respectivamente, entre universitários de ciências jurídicas e econômicas.

Analisando a diferença entre universitários dos sexos masculino e feminino em relação ao consumo de substâncias psicoativas, observa-se um perfil diferenciado entre eles. Nota-se que para as substâncias lícitas, estudantes do sexo masculino apresentaram uso maior em relação ao sexo feminino - 93,1\% dos homens fizeram uso de álcool contra $80,4 \%$ das mulheres e $41,3 \%$ dos homens fizeram uso de tabaco contra 18,9\% das mulheres. Estudo realizado por Silva et al. (2006), com universitários da área de ciências biológicas, apresenta achados semelhantes com 85,4\% de estudantes do sexo masculino contra $84,5 \%$ do sexo feminino que fizeram uso de álcool, e 24,3\% do sexo masculino contra $22 \%$ do sexo feminino que fizeram uso de tabaco.

Para as substâncias ilícitas também ocorreram diferenças entre os sexos, entre as quais o homem obteve prevalências mais altas do que as mulheres para a maconha $(20,7 \%)$, cocaína (3,4\%), anfetamínicos (24,1\%), solventes (13,8\%), ansiolíticos (13,8\%) e anticolinérgicos (3,4\%). Já, em relação aos barbitúricos e alucinógenos, o sexo masculino não apresentou uso e a prevalência entre estudantes do sexo feminino foi de 0,7\% em ambas. Em um estudo com jovens do 10 ano da Universidade Federal de Goiás (UFG), realizado por Canuto et al. (2006), encontrou-se prevalências maiores no sexo masculino para maconha, cocaína, solventes e alucinógenos e prevalências maiores no sexo feminino para ansiolíticos e anfetamínicos. Lucas et al. (2006), em uma pesquisa com universitários da área da saúde, também foram encontradas as mesmas diferenças, além de ser detectado consumo maior de barbitúricos e anticolinérgicos pelos homens.

Em nosso estudo, as anfetaminas tiveram uso maior entre os homens, resultado de certa forma inesperado, visto que tais medicamentos são muito utilizados pelas mulheres com o intuito de redução de peso. Outro achado inesperado foi o consumo maior de ansiolíticos entre os homens, provavelmente, em razão de fatores sociais inerentes a população estudada.

Quanto à idade de experimentação de substâncias psicoativas pelos estudantes, detectou-se o predomínio da faixa etária entre 16 e 18 anos para álcool, tabaco, solventes, cocaína e maconha, resultados que vão ao encontro dos achados de Fiorini et al. (2003), cujo estudo envolvendo universitários de Alfenas/MG encontrou a maioria (33\%) dos estudantes com idade de início para o uso de substâncias nessa mesma faixa etária. Observa-se, ainda, que os medicamentos anfetamínicos e ansiolíticos tiveram início mais 
tardio pela maioria dos estudantes, acima dos 18 anos, idade na qual o jovem está ingressando na faculdade. Este momento, acompanhado do estresse da nova rotina e da quantidade de atividades curriculares e extracurriculares exercidas pelos universitários são fatores que podem corroborar para o uso de tais substâncias.

Em relação ao consumo de álcool, observa-se a preferência dos estudantes pelas bebidas fermentadas - cerveja e vinho -, situação também encontrada por Balan e Campos (2006), em estudo com graduandas de Enfermagem, cuja justificativa baseia-se no fato de as bebidas fermentadas serem mais suaves que as destiladas, o fácil acesso e o baixo custo.

A maioria (36,3\%) dos estudantes relataram beber de 1 a 2 doses por vez, achado semelhante ao estudo de Rodrigues et al. (2007), envolvendo estudantes de Enfermagem, que apontou a prevalência de 31,07\% e de Chavez et al. (2005), que detectaram prevalência de 21,4\% entre universitários, ambos para a mesma quantidade de dose ingerida.

No que diz respeito ao local em que os estudantes costumam beber, a maioria $(44,1 \%)$ relataram os bares, as danceterias e as boates, sendo os amigos a companhia de maior preferência (50,8\%). Em um estudo realizado por Alves et al. (2005) com adolescentes da Bahia, verificou-se prevalência de $26,3 \%$ para os locais referidos e $54,3 \%$ para a companhia dos amigos. Nota-se que o ambiente universitário se torna favorável para o uso de bebidas alcoólicas, uma vez que há diversas comemorações, festas para receptividade de calouros, choupadas, festas para arrecadar fundos para formaturas, enfim, vários momentos atrativos para o uso do álcool, com os amigos da faculdade.

Depois do episódio de ingestão de álcool, 6,1\% dos estudantes mencionaram o envolvimento em brigas e 4,4\% dirigiram alcoolizados. Dados preocupantes, pois a agressão física pode trazer danos à pessoa alcoolizada e por em risco a vida de outras pessoas. E a associação entre uso de álcool e direção revela a necessidade de sensibilização e conscientização dos jovens sobre o risco de beber e dirigir e as conseqüências deste ato.

\section{CONCLUSÃO}

Os resultados encontrados neste estudo mostram que o consumo de substâncias psicoativas entre universitários é fator preocupante no que diz respeito à saúde dessa população, uma vez que essas substâncias são danosas ao organismo.

Sendo os estudantes de Enfermagem futuros profissionais de saúde, é de suma importância o conhecimento deles acerca da temática drogas. Sabendo que, no futuro, eles serão responsáveis por transmitir à população seus conhecimentos sobre saúde, faz-se necessário mudanças no currí- culo acadêmico, a fim de conscientizar os estudantes quanto a importância da não iniciação e da interrupção do uso indevido de drogas, alertando-os sobre as conseqüências desse hábito no âmbito biopsicossocial, bem como contribuir na formação desses profissionais, assegurando assistência de qualidade à população.

Ressaltamos, ainda, a importância de intervenções não apenas curriculares, mas também, por meio de programas preventivos destinados a essa população.

Em relação às limitações do estudo, esta pesquisa apresenta apenas uma parte da realidade dos estudantes universitários da UFES - área da saúde, o que dificulta a generalização dos dados para os demais estudantes pertencentes a outros centros universitários.

\section{AGRADECIMENTOS}

Ao Núcleo de Estudos sobre Álcool e outras Drogas (Nead) pela oportunidade de participar da pesquisa. À Fundação de Apoio à Pesquisa do Espírito Santo (Fapes) pelo suporte financeiro. A Denis Soprani Pereira, Flávia Batista Portugal e Renata Frossad Teixeira, pelo auxílio na coleta de dados.

\section{REFERÊNCIAS}

ABIPEME. Proposição para um novo critério de classificação sócio-econômica - 1978. São Paulo: Associação Brasileira dos Institutos de Pesquisa de Mercado, 1978. 15p. Mímeo.

Alves MVQMA, Costa MCO, Sobrinho CLN, Santos CAST, Gomes WA, Assis DR. Uso de bebidas alcoólicas entre adolescentes: perfil de experimentação, uso regular e fatores de risco, feira de Santana-Bahia. Revista Baiana de Saúde Pública. 29(1):91-104, 2005.

Balan TG, Campos CJG. Padrão de consumo de bebidas alcoólicas entre graduandas de enfermagem de uma Universidade Estadual Paulista. Revista Eletrônica de Saúde Mental Álcool e Drogas. 2(2):1-12, 2006.

Canuto MHA, Ferreira RA, Guimarães EMB. Uso e abuso de drogas ilícitas por jovens do 10 ano da Universidade Federal de Goiás. Revista Paulista de Pediatria. 24(2):135-42, 2006.

Carlini EA, Carlini-Cotrim B, Silva Filho AR, Barbosa MTS. II levantamento nacional sobre o uso de psicotrópicos em estudantes de primeiro e segundo graus, 1989. São Paulo: Centro Brasileiro de Informações sobre Drogas Psicotrópicas (CEBRID) - Departamento de Psicobiologia da Escola Paulista de Medicina, 1990.

Carlini EA, Galduróz JCF, Noto AR, Nappo SA. I levantamento domiciliar sobre o uso de drogas psicotrópicas no Brasil, 2001. São Paulo: Centro Brasileiro de Informações sobre Drogas Psicotrópicas (CEBRID) - Departamento de Psicobiologia da Escola Paulista de Medicina, 2001.

Carlini-Cotrim B, Carlini EA, Silva Filho AR, Barbosa MTS. 0 uso de drogas psicotrópicas por estudantes do primeiro e segundo graus da rede estadual, em dez capitais brasileiras, 1987. In: Consumo de drogas psicotrópicas no Brasil, em 1987. Centro de Documentação do Ministério da Saúde, 1989. p. 9-84. (Série C: Estudos e Projetos 5).

Carvalho AP, Rodrigues BB, Almeida J0, Macente LB. Drogas e eu com isso? Configuração do consumo de drogas entre universitários [trabalho de conclusão de curso]. Vitória: Universidade Federal do Espírito Santo. Curso de Serviço Social. Departamento de Serviço Social, 2000.

Chavez KAP, O'Brien B, Pillon SC. Uso de drogas e comportamentos de risco no contexto de uma comunidade universitária. Revista Latino-Americana de Enfermagem. 13, 2005.

Conselho Nacional de Saúde (CNS). Resolução n. 196/96 regulamenta e estabelece as diretrizes para as pesquisas envolvendo seres humanos no Brasil. [acesso em 2006 Ago 10]. Disponível em: http://www.conselho.saude.gov.br/ 
Fiorini JE, Alves AL, Ferreira LR, Fiorini CM, Durães SW, Santos RLD, et al. Use of licit and illicit drugs ate the university of Alfenas. Revista Hospital das Clínicas da Faculdade Medica de São Paulo. 58(4):199-206, 2003.

Galduróz JCF, D’Almeida V, Carvalho V, Carlini EA. III levantamento sobre 0 uso de drogas entre estudantes de primeiro e segundo graus em dez capitais brasileiras, 1993. São Paulo: Centro Brasileiro de Informações sobre Drogas Psicotrópicas (CEBRID) - Departamento de Psicobiologia da Escola Paulista de Medicina, 1994.

Galduróz JCF, Noto AR, Carlini EA. IV levantamento sobre 0 uso de drogas entre estudantes de primeiro e segundo graus em dez capitais brasileiras, 1997. São Paulo: Centro Brasileiro de Informações sobre Drogas Psicotrópicas (CEBRID) - Departamento de Psicobiologia da Escola Paulista de Medicina, 1997.

Galduróz JCF, Noto AR, Fonseca AM, Carlini EA. V levantamento nacional sobre o consumo de drogas psicotrópicas entre estudantes do ensino fundamental e médio da rede pública de ensino nas 27 capitais brasileiras, 2004. São Paulo: Centro Brasileiro de Informações sobre Drogas Psicotrópicas (CEBRID) - Departamento de Psicobiologia da Escola Paulista de Medicina, 2004.

Kerr-Correa F, Andrade AG, Bassit AZ, Boccuto NMVF. Uso de álcool e drogas por estudantes de Medicina da Unesp. Revista Brasileira de Psiquiatria. 21(2), 1999.

Lucas ACS, Parente RCP, Picanço NS, Conceição DA, Costa KRC, Magalhães IRS, et al. Uso de psicotrópicos entre universitários da área da saúde da Universidade Federal do Amazonas, Brasil. Cad. Saúde Pública. 22(3):663-71, 2006.

Matsumoto KS, Craveiro MF, Rocha PR, Lopes GT. 0 uso de tabaco entre universitários de Enfermagem da Universidade do Estado do Rio de Janeiro (UERJ). Revista Eletrônica de Saúde Mental Álcool e Drogas. 1(2):1-14, 2005.
Marçal CLA, Assis F, Lopes GT. 0 uso de bebidas alcoólicas pelos estudantes de Enfermagem da Universidade do Estado do Rio de Janeiro. Revista Eletrônica de Saúde Mental Álcool e Drogas. 2(2):1-16, 2005.

Rodrigues AP, Oliveira AS, Zaleski EGF, Arantes SL. Avaliação do nível de propensão para 0 desenvolvimento do alcoolismo entre estudantes do curso de graduação em enfermagem da Universidade Católica Dom Bosco. Revista Eletrônica de Saúde Mental Álcool e Drogas. 3(1):1-10, 2007.

Silva EF, Pavani RAB, Moraes MS, Neto FC. Prevalência do uso de drogas entre escolares do ensino médio do Município de São Jose do Rio Preto, São Paulo, Brasil. Cad. Saúde Pública. 22(6):1151, 2006

Silva LVER, Malbergier A, Stempliuk VA, Andrade AG. Fatores associados ao consumo de álcool e drogas entre estudantes universitários. Revista Saúde Pública. 40(2):280-8, 2006.

Smart RG, Anumonye A, Navaratnam V, Johnston LD, Khant U, Poshyachinda V, et al. A methodology for students drug-use surveys. Geneva: World Healh Organization, 1980.

Soldera M, Dalgalarrondo P, Corrêa Filho HR, Silva CAM. Uso de drogas psicotrópicas por estudantes: prevalência e fatores sociais associados. Revista de Saúde Pública. 38(2):277-83, 2004.

Souza FGM, Landim RM, Perdigão FB, Morais RM, Carneiro Filho BA. Consumo de drogas e desempenho acadêmico entre estudantes de medicina do Ceará. Revista de Psiquiatria Clínica. 26(4):1-11, 1999

Statistical Package Social Science - SPSS for Windows (versão 2005). Chicago : SPSS Inc., 2005.

Tavares BF, Béria JU, Lima MS. Prevalência do uso de drogas e desempenho escolar entre estudantes. Revista de Saúde Pública. 35(2):150-8, 2001. 